\title{
Eine scheinbare Abschwächung der Lokalitätsbedingung. II
}

\author{
H.-J. BORCHERS \\ Institut für Theoretische Physik der Universität Göttingen \\ K. Pohlmeyer \\ II. Institut für Theoretische Physik der Universität Hamburg \\ Received January 15, 1968
}

\begin{abstract}
If for a relativistic field theory the expectation values of the commutator $(\Psi|[A(x), A(y)]| \Psi)$ vanish in space-like direction like $\exp \left\{-\right.$ const $\left.\left|(x-y)^{2}\right|^{\alpha / 2}\right\}$ with $\alpha>1$ for sufficiently many vectors $\Psi$, it follows that $A(x)$ is a local field. Or more precisely:

For a hermitean, scalar, tempered field $A(x)$ the locality axiom can be replaced by the following conditions

1. For any natural number $n$ there exist a) a configuration $X(n)$ :

$$
X_{1}, \ldots, X_{n-1} \quad X_{1}^{i}=\cdots=X_{n-1}^{i}=0 \quad i=0,3
$$

with $\left[\sum_{i=1}^{n-2} \lambda_{i}\left(X_{i}^{1}-X_{i+1}^{1}\right)\right]^{2}+\left[\sum_{i=1}^{n-2} \lambda_{i}\left(X_{i}^{2}-X_{i+1}^{2}\right)\right]^{2}>0$ for all $\lambda_{i} \geqq 0 i=1, \ldots, n-2$, $\sum_{i=1}^{n-2} \lambda_{i}>0$, b) neighbourhoods of the $X_{i}$ 's: $U_{i}\left(X_{i}\right) \subset R^{4} i=1, \ldots, n-1$ (in the euclidean topology of $R^{4}$ ) and c) a real number $\alpha>1$ such that for all points $(x): x_{1}, \ldots, x_{n-1}: x_{i} \in U_{i}\left(X_{i}\right)$ there are positive constants $C^{(n)}\{(x)\}, h^{(n)}\{(x)\}$ with:

$\left|\left\langle\left[A\left(x_{1}\right) \ldots A\left(x_{n-1}\right), A\left(x_{n}\right)\right]\right\rangle\right|<C^{(n)}\{(x)\} \exp \left\{-h^{(n)}\{(x)\} r^{\alpha}\right\} \quad$ for $x_{n}=\left(\begin{array}{l}0 \\ 0 \\ 0 \\ r\end{array}\right), r>1$.

2. For any natural number $n$ there exist a) a configuration $Y(n)$ :

$$
Y_{2}, Y_{3}, \ldots, Y_{n} \quad Y_{3}^{i}=\cdots=Y_{n}^{i}=0 \quad i=0,3
$$

with $\left[\sum_{i=3}^{n-1} \mu_{i}\left(Y_{i}^{1}-Y_{i+1}^{1}\right)\right]^{2}+\left[\sum_{i=3}^{n-1} \mu_{i}\left(Y_{i}^{2}-Y_{i+1}^{2}\right)\right]^{2}=0 \quad$ for $\quad$ all $\quad \mu_{i} \geqq 0$, $\left.i=3, \ldots, n-1, \sum_{i=3}^{n-1} \mu_{i}>0, \quad b\right)$ neighbourhoods of the $Y_{i}^{\prime} \mathrm{s}: V_{i}\left(Y_{i}\right) \subset R^{4}$ $i=2, \ldots, n$ (in the euclidean topology of $R^{4}$ ) and c) a real number $\beta>1$ such that for all points $(y): y_{2}, \ldots, y_{n} \quad y_{i} \in V_{i}\left(Y_{i}\right)$ there are positive constants $C_{(n)}\{(y)\}$, $h_{(n)}\{(y)\}$ and a real number $\gamma_{(n)}\{(y)\} \in a$ closed subset of $R-\{0\}-\{1\}$ with: 19 Commun. math. Phys., Vol. 8
\end{abstract}


$\gamma_{(n)}\{(y)\} \cdot y_{2}, y_{3}, \ldots, y_{n}$ totally space-like in the order $2,3, \ldots, n$ and

$$
\left|\left\langle\left[A\left(x_{1}\right), A\left(x_{2}\right)\right] A\left(y_{3}\right) \ldots A\left(y_{n}\right)\right\rangle\right|<C_{(n)}\{(y)\} \exp \left\{-h_{(n)}\{(y)\} r^{\beta}\right\}
$$

for $x_{1}=\gamma_{(n)}\{(y)\} r\left(\begin{array}{l}0 \\ 0 \\ 0 \\ 1\end{array}\right), x_{2}=y_{2}-\left[1-\gamma_{(n)}\{(y)\}\right] r\left(\begin{array}{l}0 \\ 0 \\ 0 \\ 1\end{array}\right)$ and for sufficiently large values of $r$.

\section{Einleitung}

In einer vor kurzem erschienenen Arbeit [1] wurde bewiesen, daß ein hermitesches, skalares Feld $A(x)$, das die Wightman-Axiome erfüllt mit evtl. Ausnahme des Lokalitätsaxioms, strikt lokal ist, falls - grob gesprochen - die Beiträge zum Kommutator $[A(x), A(y)]$ aus dem akausalen Bereich (d. h. aus dem Bereich: $x-y$ raumartig) für große raumartige Abstände $r=\left|(x-y)^{2}\right|^{1 / 2}$ wie const. $\exp \left\{-\right.$ const $\left.^{\prime} \cdot r^{\alpha}\right\}$ mit $\alpha>2$ abfallen.

Darüber hinaus können für die Theorie einer einzigen Sorte von skalaren, neutralen Teilchen der Masse $m>0$, die mit sich selbst wechselwirken und die durch ein hermitesches, skalares, temperiertes Feld $A(x)$ beschrieben werden, mit Hilfe einer doppelten Integraldarstellung unmodifizierte Dispersionsrelationen hergeleitet werden, falls

$$
\begin{aligned}
& e^{h \sqrt{-x^{2}} \beta} \chi\left(-x^{2}\right)\left\langle p\left|\left[A\left(\frac{x}{2}\right), A\left(-\frac{x}{2}\right)\right]\right| p^{\prime}\right\rangle \in \mathscr{S}^{\prime}\left(R^{3+4+3}\right) \\
& e^{h \sqrt{-\xi_{1}^{2}} \beta} \chi\left(-\xi_{1}^{2}\right)\left\langle\left[A\left(x_{1}\right), A\left(x_{2}\right)\right]\left[A\left(x_{3}\right), A\left(x_{4}\right)\right]\right\rangle_{\substack{x_{1}-x_{2} \\
x_{3}-x_{4}=\xi_{3}}} \\
& \cdot \chi\left(-\xi_{3}^{2}\right) e^{h{\sqrt{-\xi_{3}^{2}}}^{\beta}} \in \mathscr{S}^{\prime}\left(R^{3.4}\right) \\
& \frac{x_{1}+x_{2}}{2}-\frac{x_{3}+x_{4}}{2}=\xi_{2}
\end{aligned}
$$

wobei $h>0, \beta>1$ und $\chi(t) \in C^{\infty},|\chi(t)| \leqq 1, \chi(t)=\left\{\begin{array}{l}0, t \leqq 0 \\ 1, t \geqq 1 ;\end{array}|p\rangle\right.$ und $\left|p^{\prime}\right\rangle$ sollen dabei Ein-Teilchen-Zustände zu den Impulsen $p$ und $p^{\prime}$ bezeichnen.

Dieses letztere Resultat legt die Vermutung nahe, daß die Schranke 2 für die exponentielle Ordnung des Abfalls der Beiträge zum Kommutator $[A(x), A(y)]$ aus dem akausalen Bereich nicht optimal ist, sondern sich auf 1 verbessern lassen sollte.

In der hier vorliegenden Arbeit soll die Richtigkeit dieser Vermutung zunächst am Beispiel der ${ }^{1}$ 3-Punkt-Funktion, dann aber in voller Allgemeinheit gezeigt werden. In einem ersten Schritt werden in den Vakuumerwartungswerten zyklische Umstellungen der Feldoperatoren

1 Bekanntlich liefert die Lokalität keine Einschränkung für die 2-PunktFunktion eines hermiteschen, skalaren Feldes. 
betrachtet, was auf eine Vergrößerung der Regularitätsgebiete führt. Die so vergrößerten Regularitätsgebiete erlauben, aus einem Abfall des Kommutators für große raumartige Abstände $r$ wie const. $\cdot \exp \left\{\right.$ - const' $\left.\cdot r^{\beta}\right\}$ mit $\beta>1$ dann in einem zweiten Schritt strikte Lokalität zu schließen.

Für die 3-Punkt-Funktion selbst werden wir ein etwas schärferes Resultat erhalten.

\section{Dreipunktfunktion}

Wir gehen aus von den reellen Punkten $x_{1}, x_{2}, x_{3}$, die in dem Durchschnitt der "extended tubes" der 3-Punkt-Funktionen

$$
\begin{aligned}
W_{123}\left(\begin{array}{l}
\left(x_{1}-x_{2}\right)^{2} \\
\left(x_{2}-x_{3}\right)^{2} \\
\left(x_{3}-x_{1}\right)^{2}
\end{array}\right) & =\left\langle A\left(x_{1}\right) A\left(x_{2}\right) A\left(x_{3}\right)\right\rangle \text { und } W_{312}\left(\begin{array}{c}
\left(x_{1}-x_{2}\right)^{2} \\
\left(x_{2}-x_{3}\right)^{2} \\
\left(x_{3}-x_{1}\right)^{2}
\end{array}\right) \\
& =\left\langle A\left(x_{3}\right) A\left(x_{1}\right) A\left(x_{2}\right)\right\rangle
\end{aligned}
$$

liegen. Wie R. Jost gezeigt hat [2] sind das gerade die in der Reihenfolge 1, 2, 3 bzw. 3, 1, 2 total raumartigen Punkte ${ }^{2}$. Durch reelle Lorentztransformationen lassen sich solche Punkte stets auf die folgende Gestalt bringen:

$$
x_{1}=\left(\begin{array}{l}
0 \\
x_{1}^{1} \\
0 \\
0
\end{array}\right) x_{2}=\left(\begin{array}{c}
0 \\
-x_{1}^{1} \\
0 \\
0
\end{array}\right) x_{3}=\left(\begin{array}{l}
0 \\
x_{3}^{2} \\
x_{3}^{2} \\
0
\end{array}\right), x_{1}^{1}, x_{3}^{2}>0
$$

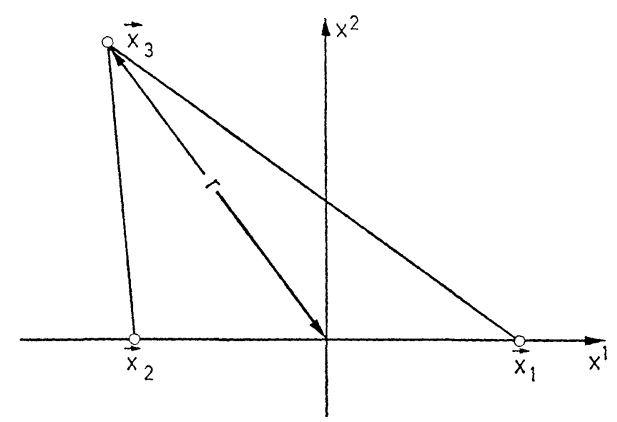

Fig. 1

$$
\begin{aligned}
& z_{1}=\left(x_{1}-x_{2}\right)^{2}=z_{2}^{\prime}=-4\left(x_{1}^{1}\right)^{2} \\
& z_{2}=\left(x_{2}-x_{3}\right)^{2}=z_{3}^{\prime}=-\left(x_{1}^{1}\right)^{2}-2 x_{1}^{1} x_{3}^{1}-r^{2} \\
& z_{3}=\left(x_{3}-x_{1}\right)^{2}=z_{1}^{\prime}=-\left(x_{1}^{1}\right)^{2}+2 x_{1}^{1} x_{3}^{1}-r^{2} .
\end{aligned}
$$

${ }^{2}$ Die hier als $(1,2,3)$-total raumartig benannten Punkte werden auch als $(1,2,3)$-Jost Punkte bezeichnet. 
Wir benutzen nun die von G. KäLlén und A. S. Wightman explizit angegebene Charakterisierung der "extended tubes" der 3-Punkt-Funktion in den Invarianten [3] und finden, daß

$$
\left\{W_{123}-W_{312}\right\}\left(\left(\begin{array}{l}
-4\left(x_{1}^{1}\right)^{2} \\
-\left(x_{1}^{1}\right)^{2}-2 x_{1}^{1} x_{3}^{1} \\
-\left(x_{1}^{1}\right)^{2}+2 x_{1}^{1} x_{3}^{1}
\end{array}\right)+z\left(\begin{array}{r}
0 \\
-1 \\
-1
\end{array}\right)\right)
$$

eine in der längs der reellen Achse von $-\infty$ bis $+\left(x_{3}^{1}\right)^{2}$ aufgeschnittenen $z$-Ebene reguläre Funktion ist bzw. daß für alle reellen $x_{1}^{1}, x_{3}^{1},: x_{1}^{1}>0$,

$$
\left\{W_{123}-W_{312}\right\}\left(-\left(\begin{array}{l}
4\left(x_{1}^{1}\right)^{2} \\
\left(x_{1}^{1}+x_{3}^{1}\right)^{2} \\
\left(x_{1}^{1}-x_{3}^{1}\right)^{2}
\end{array}\right)+w^{2}\left(\begin{array}{r}
0 \\
-1 \\
-1
\end{array}\right)\right)
$$

eine in der Halbebene $\operatorname{Re} w>0$ reguläre und wegen der Temperiertheit des Feldes $A(x)$ dort auch polynomial beschränkte Funktion ist.

Wenn wir nun voraussetzen, daß es eine Eindeutigkeitsmenge $E_{[12,3]}$ $\bar{E}_{[12,3]} \subset(R-\{0\}) \times R$ gibt (z. B. $E_{[12,3]}=\left\{\left(x_{1}^{1}, x_{3}^{1}\right) / x_{1}^{1}=\wp_{1}^{1} \cdot(1+1 / m)\right.$, $\left.\left.x_{3}^{1}=\grave{x}_{3}^{1}+1 / n, \quad m, n=1,2, \ldots ; \grave{x}_{1}^{1}>0\right\}\right)$ derart, daß für alle $\left(x_{1}^{1}, x_{3}^{1}\right)$ $\in E_{[12,3]}$ :

wobei

$$
\limsup _{r \rightarrow+\infty} \frac{\log \left|\left\langle\left[A\left(x_{1}\right) A\left(x_{2}\right), A\left(x_{3}\right)\right]\right\rangle\right|}{r}=-\infty
$$

$$
x_{1}=\left(\begin{array}{l}
0 \\
x_{1}^{1} \\
0 \\
0
\end{array}\right) \quad x_{2}=\left(\begin{array}{c}
0 \\
-x_{1}^{1} \\
0 \\
0
\end{array}\right) \quad x_{3}=\left(\begin{array}{l}
0 \\
x_{3}^{1} \\
\sqrt{r^{2}-\left(x_{3}^{1}\right)^{2}} \\
0
\end{array}\right)_{r>\left|x_{3}^{1}\right|}, \quad x_{1}^{1} \neq 0,
$$

dann folgt $\left\{W_{123}-W_{312}\right\}\left(\begin{array}{l}z_{1} \\ z_{2} \\ z_{3}\end{array}\right) \equiv 0$ in (der) Zusammenhangskomponenten (Zusammenhangskomponente) $K_{[12,3]}$ von $I\left(\mathscr{T}_{123}^{\prime} \cap \mathscr{T}_{312}^{\prime}\right)$, welche (die) in den Reihenfolgen 1, 2, 3 und 3,1,2 total raumartige(n) reelle(n) Punkte als innere Punkte enthalten (enthält). Dabei soll $I$ die Abbildung auf die Invarianten bezeichnen.

Es gibt nämlich keine in der Halbebene $\mathrm{Re} w>0$ reguläre, polynomial beschränkte Funktion $f(w) \neq 0$ mit

$$
\limsup _{w, \text { reell } \rightarrow+\infty} \frac{\log |f(w)|}{w}=-\infty \text {. }
$$

(Der Beweis wird im Anhang erbracht.) Daher gilt:

$$
\left\{W_{123}-W_{312}\right\}\left(-\left(\begin{array}{l}
4\left(x_{1}^{1}\right)^{2} \\
\left(x_{1}^{1}+x_{3}^{1}\right)^{2} \\
\left(x_{1}^{1}-x_{3}^{1}\right)^{2}
\end{array}\right)+w^{2}\left(\begin{array}{r}
0 \\
-1 \\
-1
\end{array}\right)\right) \equiv 0
$$

für $\operatorname{Re} w>0$ und alle $\left(x_{1}^{1}, x_{3}^{1}\right) \in E_{[12,3]}$. Da laut Voraussetzung $E_{[12,3]}$ eine Eindeutigkeitsmenge ist, ergibt sich wie behauptet $\left\{W_{123}-W_{312}\right\}\left(\begin{array}{l}z_{1} \\ z_{2} \\ z_{3}\end{array}\right) \equiv 0$ in $K_{[12,3]}\left\{I\left(\mathscr{T}_{123}^{\prime} \cap \mathscr{T}_{312}^{\prime}\right)\right\}$ und insbesondere : 
$\left\langle\left[A\left(x_{1}\right) A\left(x_{2}\right), A\left(x_{3}\right)\right]\right\rangle \equiv 0$ für alle reellen Punkte $x_{1}, x_{2}, x_{3}$, die sowohl in der Reihenfolge 1, 2, 3 wie auch in der Reihenfolge 3, 1, 2 total raumartig sind. Indem wir die angeführten Argumente für $\left\{W_{312}-W_{231}\right\}$ wiederholen, können wir schließen:

$\left\{W_{312}-W_{231}\right\}\left(\begin{array}{l}z_{1} \\ z_{2} \\ z_{3}\end{array}\right) \equiv 0$ in $K_{[31,2]}\left\{I\left(\mathscr{T}_{312}^{\prime} \cap \mathscr{T}_{231}^{\prime}\right)\right\}$ und insbesondere: $\left\langle\left[A\left(x_{3}\right) A\left(x_{1}\right), A\left(x_{2}\right)\right]\right\rangle \equiv 0$ für alle reellen Punkte $x_{1}, x_{2}, x_{3}$, die sowohl in der Reihenfolge 3, 1, 2 wie auch in der Reihenfolge 2, 3, 1 total raumartig sind. $W_{123}, W_{312}$ und $W_{231}$ sind also Teile einer einzigen in der Holomorphiehülle $\mathscr{H}^{3}$ von $I\left(\mathscr{T}_{123}^{\prime}\right) \cap I\left(\mathscr{T}_{312}^{\prime}\right) \cap I\left(\mathscr{T}_{231}^{\prime}\right)$ regulären Funktion $W_{123}^{z y k l}\left(\begin{array}{l}z_{1} \\ z_{2} \\ z_{3}\end{array}\right)$. Ebenso sind $W_{213}, W_{321}$ und $W_{132}$ Teile einer einzigen in der Holomorphiehülle $\mathscr{H}^{\prime 4}$ von $I\left(\mathscr{T}_{213}^{\prime}\right) \cap I\left(\mathscr{T}_{321}^{\prime}\right) \cap I\left(\mathscr{T}_{132}^{\prime}\right)$ regulären Funktion $W_{213}^{\mathrm{zykl}}\left(\begin{array}{c}z_{1} \\ z_{2} \\ z_{3}\end{array}\right)$.

Als nächstes betrachten wir die reellen Punkte $x_{1}, x_{2}, x_{3}$, die in dem Durchschnitt der "extended tubes" $\mathscr{T}_{123}^{\prime}$ und $\mathscr{T}_{213}^{\prime}$ liegen. Das sind gerade die in den Reihenfolgen 1, 2, 3 und 2, 1, 3 total raumartigen Punkte [2]. Durch reelle Lorentztransformationen lassen sich solche Punkte stets auf die folgende Gestalt bringen:

$$
x_{1}=\left(\begin{array}{l}
0 \\
x_{1}^{1} \\
0 \\
0
\end{array}\right) \quad x_{2}=\left(\begin{array}{c}
0 \\
x_{2}^{1} \\
0 \\
0
\end{array}\right) \quad x_{3}=\left(\begin{array}{l}
0 \\
0 \\
x_{3}^{2} \\
0
\end{array}\right) \quad x_{1}^{1}>x_{2}^{1}, x_{3}^{2}>0 .
$$

Wir interessieren uns speziell für solche Punkte, die sich auf die Gestalt $x_{1}=\left(\begin{array}{l}0 \\ x_{1}^{1} \\ 0 \\ 0\end{array}\right) x_{2}=\left(\begin{array}{l}0 \\ x_{2}^{1} \\ 0 \\ 0\end{array}\right) x_{3}=\left(\begin{array}{l}0 \\ 0 \\ x_{3}^{2} \\ 0\end{array}\right) x_{1}^{1}>0, x_{3}^{2}>0, x_{2}^{1}<0, \frac{x_{1}^{1}}{\left|x_{2}^{1}\right|}=\frac{\gamma}{1-\gamma}, 0<\gamma<1$

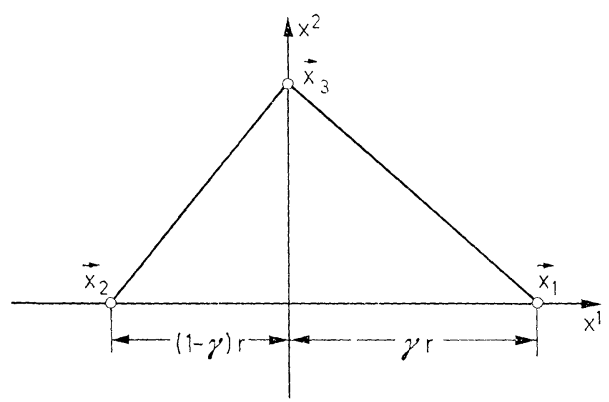

Fig. 2

3,4 Evtl. nicht schlicht 
bringen lassen:

$$
\begin{aligned}
& z_{1}=\left(x_{1}-x_{2}\right)^{2}=z_{1}^{\prime}=-r^{2} \\
& z_{2}=\left(x_{2}-x_{3}\right)^{2}=z_{3}^{\prime}=-(1-\gamma)^{2} \cdot r^{2}-\left(x_{3}^{2}\right)^{2} \\
& z_{3}=\left(x_{3}-x_{1}\right)^{2}=z_{2}^{\prime}=-\gamma^{2} \cdot r^{2}-\left(x_{3}^{2}\right)^{2} .
\end{aligned}
$$

Wir benutzen wiederum die von KäLLÉN und WIGHTMAN explizit angegebene Charakterisierung der "extended tubes" der 3-Punkt-Funktion in den Invarianten [3] und können so unter Ausnutzung der Regularitätsgebiete $I\left(\mathscr{T}_{231}^{\prime}\right)$ bzw. $I\left(\mathscr{T}_{132}^{\prime}\right)$ zeigen, daß für alle $x_{3}^{2}>0,0<\gamma<1$ :

$$
\left\{W_{123}^{\mathrm{zykl}}-W_{213}^{\mathrm{zykl}}\right\}\left(-\left(\begin{array}{l}
0 \\
\left(x_{3}^{2}\right)^{2} \\
\left(x_{3}^{2}\right)^{2}
\end{array}\right)+z\left(\begin{array}{l}
-1 \\
-(1-\gamma)^{2} \\
-\gamma^{2}
\end{array}\right)\right)
$$

eine in der längs der reellen Achse von $-\infty$ bis 0 aufgeschnittenen $z$-Ebene reguläre Funktion ist.

Die Transformation $\begin{cases}\zeta_{1}=z_{1} & \zeta_{2}=\frac{z_{1}-z_{2}+z_{3}}{2 z_{1}} \zeta_{3}=\frac{\left[z_{1}-z_{2}+z_{3}\right]^{2}}{4 z_{1}}-z_{3} \\ z_{1}=\zeta_{1} & z_{2}=\zeta_{1}\left[1-\zeta_{2}\right]^{2}-\zeta_{3} \quad z_{3}=\zeta_{1} \zeta_{2}^{2}-\zeta_{3}\end{cases}$ ist für $z_{1} \neq 0$ bi-regulär. Daher ist:

$$
\left\{W_{123}^{\mathrm{zykl}}-W_{213}^{\mathrm{zykl}}\right\}\left(\zeta_{1}, \zeta_{2}, \zeta_{3}\right)_{/ \zeta_{1}=-w^{2}}
$$

in $\{w / \operatorname{Re} w>0\} \times\left\{\zeta_{2} / \zeta_{2}\right.$ reell, $\left.0<\zeta_{2}<1\right\} \times\left\{\zeta_{3} / \zeta_{3}\right.$ reell, $\left.>0\right\}$ regulär und dort für feste reelle $\zeta_{2}, \zeta_{3}: 0<\zeta_{2}<1,0<\zeta_{3}$ auch polynomial beschränkt.

Setzen wir nun voraus, daß es eine Eindeutigkeitsmenge $E_{[1,2]_{3}}$ : $\left.: \bar{E}_{[1,2]_{3}} \subset\right] 0,1[\times] 0,+\infty\left[\right.$ gibt, derart daß für alle $\left[\left(\gamma,\left(x_{3}^{2}\right)^{2}=\right]\right.$ $\left(\zeta_{2}, \zeta_{3}\right) \in E_{[1,2]_{3}}$ :

wobei

$$
\limsup _{r \rightarrow+\infty} \frac{\log \left|\left\langle\left[A\left(x_{1}\right), A\left(x_{2}\right)\right] A\left(x_{3}\right)\right\rangle\right|}{r}=-\infty,
$$

$$
x_{1}=\gamma r\left(\begin{array}{l}
0 \\
1 \\
0 \\
0
\end{array}\right) \quad x_{2}=-(1-\gamma) r\left(\begin{array}{l}
0 \\
1 \\
0 \\
0
\end{array}\right) \quad x_{3}=x_{3}^{2}\left(\begin{array}{l}
0 \\
0 \\
1 \\
0
\end{array}\right), \quad x_{3}^{2}>0,
$$

dann folgt $\left\{W_{123}^{z y k l}-W_{213}^{\text {zykl }}\right\}\left(\begin{array}{c}z_{1} \\ z_{2} \\ z_{3}\end{array}\right) \equiv 0$ in (der) Zusammenhangskomponenten (Zusammenhangskomponente) von $\mathscr{H}\left\{I\left(\mathscr{T}_{123}^{\prime}\right) \cup I\left(\mathscr{T}_{312}^{\prime}\right) \cup I\left(\mathscr{T}_{231}^{\prime}\right)\right\}$ $\cap \mathscr{H}^{\prime}\left\{I\left(\mathscr{T}_{213}^{\prime}\right) \cup I\left(\mathscr{T}_{321}^{\prime}\right) \cup I\left(\mathscr{T}_{132}^{\prime}\right)\right\}$, welche (die) in den Reihenfolgen 1, 2, 3 und 2, 1, 3 total raumartige(n) reelle(n) Punkte als innere Punkte enthalten (enthält), insbesondere also sowohl in den reellen Punkten, die in den Reihenfolgen 1, 2,3 und 2, 1,3 total raumartig sind, als auch in den reellen Punkten, die in den Reihenfolgen 1,2,3 und 1,3,2 total raumartig sind. 
Wir brauchen nur noch ein Argument von Wightman [5] anzuwenden, um zu zeigen, daß für alle reellen Punkte $x_{1}, x_{2}, x_{3}$ gilt:

$$
\left\langle\left[A\left(x_{1}\right), A\left(x_{2}\right)\right] A\left(x_{3}\right)\right\rangle=0 \text { falls } \quad\left(x_{1}-x_{2}\right)^{2}<0
$$

bzw.

$$
\left\langle A\left(x_{1}\right)\left[A\left(x_{2}\right), A\left(x_{3}\right)\right]\right\rangle=0 \text { falls }\left(x_{2}-x_{3}\right)^{2}<0 .
$$

Wir fassen die obigen Überlegungen in dem folgenden Satz zusammen:

Satz 1. Sei $A(x)$ ein hermitesches, skalares Feld, das die Wightman Axiome erfüllt mit evtl. Ausnahme des Lokalitätsaxioms, welches wir durch die folgende Voraussetzung ersetzen wollen:

Es gibt zwei Eindeutigkeitsmengen $E_{[12,3]}: \bar{E}_{[12,3]} \subset(R-\{0\}) \times R$ und $\left.E_{[1,2]_{3}}: \bar{E}_{[1,2]_{3}} \subset\right] 0,1[\times] 0,+\infty\left[\right.$ derart, daß für alle $\left(x_{1}^{1}, x_{3}^{1}\right) \in E_{[12,3]}$ :

wobei

$$
\limsup _{r \rightarrow+\infty} \frac{\log \left|\left\langle\left[A\left(x_{1}\right) A\left(x_{2}\right), A\left(x_{3}\right)\right]\right\rangle\right|}{r}=-\infty,
$$

$$
x_{1}=\left(\begin{array}{l}
0 \\
x_{1}^{1} \\
0 \\
0
\end{array}\right) \quad x_{2}=\left(\begin{array}{c}
0 \\
-x_{1}^{1} \\
0 \\
0
\end{array}\right) \quad x_{3}=\left(\begin{array}{l}
0 \\
x_{3}^{1} \\
\sqrt{r^{2}-\left(x_{3}^{1}\right)^{2}} \\
0
\end{array}\right)_{r>\left|x_{3}^{1}\right|},
$$

bzw. für alle $\left(\gamma,\left(x_{3}^{2}\right)^{2}\right) \in E_{[1,2] 3}$ :

wobei

$$
\limsup _{r \rightarrow+\infty} \frac{\log \left|\left\langle\left[A\left(x_{1}\right), A\left(x_{2}\right)\right] A\left(x_{3}\right)\right\rangle\right|}{r}=-\infty,
$$

$$
x_{1}=\left(\begin{array}{l}
0 \\
\gamma r \\
0 \\
0
\end{array}\right) \quad x_{2}=\left(\begin{array}{l}
0 \\
-(1-\gamma) r \\
0 \\
0
\end{array}\right) \quad x_{3}=\left(\begin{array}{l}
0 \\
0 \\
x_{3}^{2} \\
0
\end{array}\right) .
$$

Dann gilt für alle reellen Punkte $x_{1}, x_{2}, x_{3}$ mit $\left(x_{1}-x_{2}\right)^{2}<0$ :

$$
\left\langle\left[A\left(x_{1}\right), A\left(x_{2}\right)\right] A\left(x_{3}\right)\right\rangle=0
$$

und für alle reellen Punkte $x_{1}, x_{2}, x_{3}$ mit $\left(x_{2}-x_{3}\right)^{2}<0$ :

$$
\left\langle A\left(x_{1}\right)\left[A\left(x_{2}\right), A\left(x_{3}\right)\right]\right\rangle=0 .
$$

Für echt nicht-lokale 3-Punkt-Funktionen sind also

$$
\begin{gathered}
M\left(x_{1}^{1}, x_{3}^{1}\right)=-\limsup _{r \rightarrow+\infty} \frac{\log \left|\left\langle\left[A\left(x_{1}\right) A\left(x_{2}\right), A\left(x_{3}\right)\right]\right\rangle\right|}{r} \\
x_{1}=\left(\begin{array}{c}
0 \\
x_{1}^{1} \\
0 \\
0
\end{array}\right) \quad x_{2}=\left(\begin{array}{c}
0 \\
-x_{1}^{1} \\
0 \\
0
\end{array}\right) \quad x_{3}=\left(\begin{array}{l}
0 \\
x_{3}^{1} \\
\sqrt{r^{2}-\left(x_{3}^{1}\right)^{2}}
\end{array}\right)_{r>\left|x_{3}^{1}\right|} \quad\left(x_{1}^{1}, x_{3}^{1}\right) \in E_{[12,3]}
\end{gathered}
$$


und

$$
\begin{gathered}
M^{\prime}\left(\gamma,\left(x_{3}^{2}\right)^{2}\right)=-\limsup _{r \rightarrow+\infty} \frac{\log \left|\left\langle\left[A\left(x_{1}\right), A\left(x_{2}\right)\right] A\left(x_{3}\right)\right\rangle\right|}{r} \\
x_{1}=\left(\begin{array}{l}
0 \\
\gamma r \\
0 \\
0
\end{array}\right) \quad x_{2}=\left(\begin{array}{l}
0 \\
0 \\
0 \\
0
\end{array}\right) \quad x_{3}=\left(\begin{array}{l}
0 \\
0 \\
x_{3}^{2} \\
0
\end{array}\right) \quad\left(\gamma,\left(x_{3}^{2}\right)^{2}\right) \in E_{\lceil 1,2] 3},
\end{gathered}
$$

nicht beide gleichzeitig $\equiv+\infty$. Die Infima:

$$
\inf _{x_{1}^{1}, x_{3}^{1}, E_{[12,3]}} \frac{M\left(x_{1}^{1}, x_{1}^{1}\right) /\left(x_{1}^{1} x_{3}^{1}\right) \in E_{[12,3]}}{m} \inf _{\gamma,\left(x_{3}^{2}\right)^{2}, E_{[1,2] 3}} \frac{M^{\prime}\left(\gamma,\left(x_{3}^{2}\right)^{2}\right) /\left(\gamma,\left(x_{3}^{2}\right)^{2}\right) \in E_{[1,223}}{m} .
$$

können aber beliebig groß sein wie das Beispiel: $A(x)=A_{0}(x)+: A_{0}^{2}:(x)$ $+: A_{0}^{2 n}:(x)+A_{0}^{-}(x)^{2 n} \cdot A_{0}^{+}(x)^{2 n}$ zeigt. Dabei ist $A_{0}(x)=A_{0}^{+}(x)+A_{0}^{-}(x)$ ein freies Feld zur Masse $m>0$ und $n$ eine beliebige natürliche Zahl.

\section{Allgemeine $n$-Punkt-Funktion}

$B_{n}(1)\left\{\begin{array}{l}\text { Es gebe a) eine Konfiguration }(X): X_{1}=\left(\begin{array}{l}0 \\ X_{1}^{1} \\ X_{1}^{2} \\ 0\end{array}\right), \ldots, X_{n-1}==\left(\begin{array}{l}0 \\ X_{n-1}^{1} \\ X_{n-1}^{2} \\ 0\end{array}\right) \\ \text { mit }\left[\sum_{i=1}^{n-2} \lambda_{i}\left(X_{i}^{1}-X_{i+1}^{1}\right]+\left[\sum_{i=1}^{n-2} \lambda_{i}\left(X_{i}^{2}-X_{i+1}^{2}\right)\right]^{2}>0 \text { für alle }\right. \\ \left.\lambda_{i} \geqq 0 i=1, \ldots, n-2, \sum_{i=1}^{n-2} \lambda_{i}>0, b\right) \text { Umgebungender } X_{i}: U_{i}\left(X_{i}\right) \subset R^{4} \\ \left.i=1, \ldots, n-1 \text { (in der euklidischen Topologie des } R^{4}\right) \text { und } \\ \text { c) eine reelle Zahl } \alpha>1 \text { derart, daß für alle Punkte }(x): \\ x_{1}, \ldots, x_{n-1} x_{i} \in U_{i}\left(X_{i}\right) \text { positive Konstanten } C^{(n)}\{(x)\}, h^{(n)}\{(x)\} \\ \text { existieren mit: } \\ \left.K\left[A\left(x_{1}\right) \ldots A\left(x_{n-1}\right), A\left(x_{n}\right)\right]\right\rangle \mid<C^{(n)}\{(x)\} \exp \left\{-h^{(n)}\{(x)\} \cdot r^{\alpha}\right\} \\ \text { für } x_{n}=\left(\begin{array}{l}0 \\ 0 \\ 0 \\ r\end{array}\right), r>1 .\end{array}\right.$

Dann folgt für eine beliebige Permutation $\pi$ von $1, \ldots, n$, da $\beta$ die $W^{\sigma^{k} \pi}\left(\zeta_{1}, \ldots, \zeta_{n-1}\right)=\mathscr{L}\left\{\widetilde{W}\left(p_{1}, \ldots, p_{n-1}\right)\right\}^{\sigma^{k} \pi}\left(\zeta_{1}, \ldots, \zeta_{n-1}\right) k=0, \ldots$,

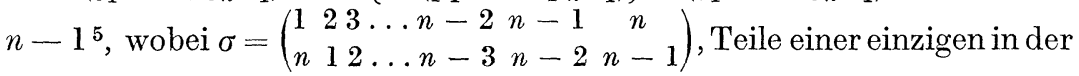
Holomorphiehülle $\mathscr{H}^{6}$ von $\bigcup_{k=0}^{n-1} \mathscr{T}_{\sigma k_{\pi}\{1, \ldots, n\}}^{\prime}$ regulären Funktion $W_{\pi\{1, \ldots, n\}}^{\mathrm{zykl}}\left(\zeta_{1}, \ldots, \zeta_{n-1}\right)$ sind.

${ }^{5} \mathscr{L}$ bezeichne die Laplace Transformierte.

${ }^{6}$ Evtl. nicht schlicht. 


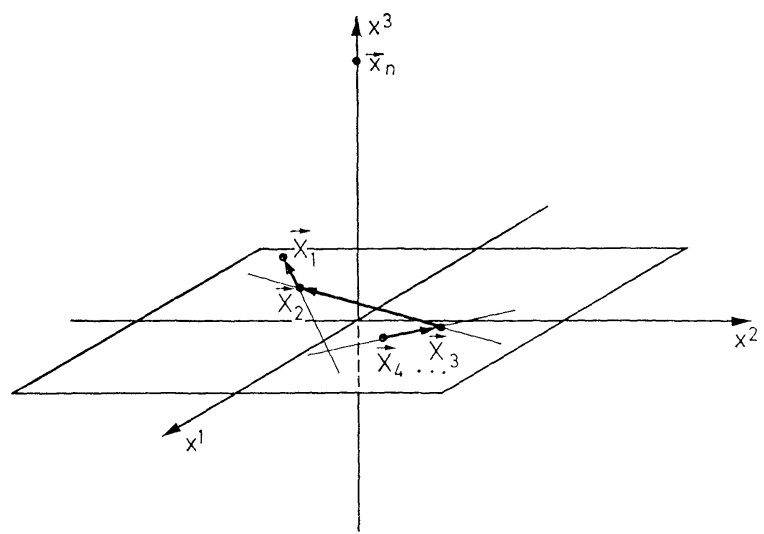

Fig. 3

Beweis. Ohne Beschränkung der Allgemeinheit dürfen wir annehmen: $\pi=\mathrm{id}$. Wir betrachten: $W\left(\zeta_{1}, \ldots, \zeta_{n-1}\right)-W^{\sigma}\left(\zeta_{1} \ldots, \zeta_{n-1}\right)$

$$
=\left\{W_{12 \ldots(n-1) n}-W_{n 1 \ldots(n-2)(n-1)}\right\}\left(\zeta_{1}, \ldots, \zeta_{n-1}\right) \text {. }
$$

$\mathrm{Zu}$ jedem $\varepsilon, \frac{\pi}{2 \alpha}(\alpha-1)>\varepsilon>0$ gibt es Umgebungen $V_{i}^{\varepsilon}\left(X_{i}\right) \subset U_{i}\left(X_{i}\right)$, so $\mathrm{da} B$

$$
\left\{W_{12 \ldots(n-1) n}-W_{n 1 \ldots(n-2)(n-1)}\right\}\left(x_{1}-x_{2}, \ldots, x_{n-2}-x_{n-1}, x_{n-1}-\left(\begin{array}{l}
0 \\
0 \\
0 \\
w
\end{array}\right)\right)
$$

$=W_{\left[12 \ldots(n-1),{ }_{n]}\right.}((x) ; w)$ in dem Winkelbereich $-\frac{\pi}{2 \alpha}-\varepsilon \leqq \arg (w-1)$ $\leqq \frac{\pi}{2 \alpha}+\varepsilon$ regulär ist. Wenn wir nun zu den Variablen $z=(w-1)^{\alpha}$ übergehen, entsteht folgende Situation:

$W_{[12, \ldots(n-1), n]}((x) ; w)=W^{[12 \ldots(n-1), n]}((x) ; z)$ ist in dem Winkelbereich $-\alpha \varepsilon-\frac{\pi}{2} \leqq \Theta=\arg z \leqq \frac{\pi}{2}+\alpha \varepsilon$ regulär und dort von exponentiellem Typ (sogar polynomial beschränkt)

$$
\limsup _{|z| \rightarrow+\infty} \frac{\log \left|W^{[12 \ldots(n-1), n]}\left((x) ;|z| e^{i \Theta}\right)\right|}{|z|}= \begin{cases}=0 & \text { für } \Theta=-\alpha \varepsilon-\pi / 2 \\ =0 & \text { für } \Theta=\alpha \varepsilon+\pi / 2 \\ <0 & \text { für } \Theta=0\end{cases}
$$

Nach einem Theorem über Funktionen von exponentiellem Typ [6] muß notwendig gelten:

$$
W^{[12 \ldots(n-1), n]}((x) ; z) \equiv 0 \quad \text { in } \quad-\alpha \varepsilon-\frac{\pi}{2} \leqq \arg z \leqq \frac{\pi}{2}+\alpha \varepsilon
$$


und insbesondere

$\left\{W_{12 \ldots(n-1) n}-W_{n 1 \ldots(n-2)(n-1)}\right\}\left(x_{1}-x_{2}, \ldots, x_{n-2}-x_{n-1}, x_{n-1}\right.$

$\left.-\left(\begin{array}{l}0 \\ 0 \\ 0 \\ x_{n}^{3}\end{array}\right)\right) \equiv 0, x_{n}^{3} \geqq 1$. Daraus ergibt sich:

$$
\left\{W_{12 \ldots(n-1) n}-W_{n 1 \ldots(n-2)(n-1)}\right\}\left(\zeta_{1}, \ldots, \zeta_{n-1}\right) \equiv 0,
$$

in (der) Zusammenhangskomponenten (Zusammenhangskomponente) $K_{[12 \ldots(n-1), n]}$ von $\mathscr{T}_{12 \ldots(n-1) n}^{\prime} \cap \mathscr{T}_{n 1 \ldots(n-2)(n-1)}^{\prime}$ welche (die) in den Reihenfolgen $1,2, \ldots,(n-1), n$ und $n, 1, \ldots,(n-2),(n-1)$ total raumartige (n) Punkte als innere Punkte enthalten (enthält). Dieses Argument wiederholen wir für

$$
\left\{W_{\sigma^{k}\{1, \ldots, n\}}-W_{\sigma^{k+1}\{1, \ldots, n\}}\right\}\left(\zeta_{1}, \ldots, \zeta_{n-1}\right) \quad k=1, \ldots, n-2
$$

und erhalten auf diese Weise, daß die $W \sigma^{k}$.id $\left(\zeta_{1}, \ldots, \zeta_{n-1}\right) k_{n-1}=0,1, \ldots$, $n-1$ Teile einer einzigen in der Holomorphiehülle $\mathscr{H}$ von $\bigcup_{k=0}^{n-1} \mathscr{T}_{\sigma}{ }^{k}\{1, \ldots, n\}$ regulären Funktion $W_{\mathrm{id}\{1, \ldots, n\}}^{z y \mathrm{kl}}\left(\zeta_{1}, \ldots, \zeta_{n-1}\right)$ sind. $\quad$ q.e.d.

Im folgenden wollen wir weiter voraussetzen:

$$
B_{n}(2)\left\{\begin{array}{l}
\text { Es gebe a) eine Konfiguration } Y(n): Y_{2}, Y_{3}=\left(\begin{array}{l}
0 \\
Y_{3}^{1} \\
Y_{3}^{2} \\
0
\end{array}\right), \ldots, Y_{n}=\left(\begin{array}{l}
0 \\
Y_{n}^{1} \\
Y_{n}^{1} \\
0
\end{array}\right) \\
\text { mit }\left[\sum_{i=3}^{n-1} \mu_{i}\left(Y_{i}^{1}-Y_{i+1}^{1}\right)\right]^{2}+\left[\sum_{i=3}^{n-1} \mu_{i}\left(Y_{i}^{2}-Y_{i+1}^{2}\right)\right]^{2}>0 \text { für alle } \\
\left.\mu_{i} \geqq 0 i=3, \ldots, n-1, \sum_{i=3}^{n-1} \mu_{i}>0, \mathrm{~b}\right) \text { Umgebungen der } Y_{i}: V_{i}\left(Y_{i}\right) \\
\left.\mathcal{L} R^{4} i=2, \ldots, n \text { (in der euklidischen Topologie des } R^{4}\right) \text { und } \\
\text { c) eine reelle Zahl } \beta>1 \text { derart, daß für alle Punkte }(y): y_{2}, \ldots, y_{n} \\
y_{i} \in V_{i}\left(Y_{i}\right) \text { positive Konstanten } C_{(n)}\{(y)\}, h_{(n)}\{(y)\} \text { und eine reelle } \\
\text { Zahl } \gamma_{(n)}\{(y)\} \in \text { einer abgeschlossenen Untermenge von } R-\{0\} \\
-\{1\} \text { existieren mit: } \gamma(n)\{(y)\} y_{2}, y_{3}, \ldots, y_{n} \text { total raumartig in } \\
\text { der Reihenfolge } 2,3, \ldots, n \text { und } \\
\left.K\left[A\left(x_{1}\right), A\left(x_{2}\right)\right] A\left(y_{3}\right) \ldots A\left(y_{n}\right)\right\rangle \mid<C_{(n)}\{(y)\} \text { exp }\left\{-h_{(n)}\{(y)\} \cdot r^{\beta}\right\} \\
\text { für } x_{1}=\gamma(n)\{(y)\} r\left(\begin{array}{l}
0 \\
0 \\
0 \\
l
\end{array}\right), x_{2}=y_{2}-\left[1-\gamma_{(n)}\{(y)\}\right] r\left(\begin{array}{l}
0 \\
0 \\
0 \\
l
\end{array}\right) \text { und alle } \\
\text { hinreichend großen } r .
\end{array}\right.
$$




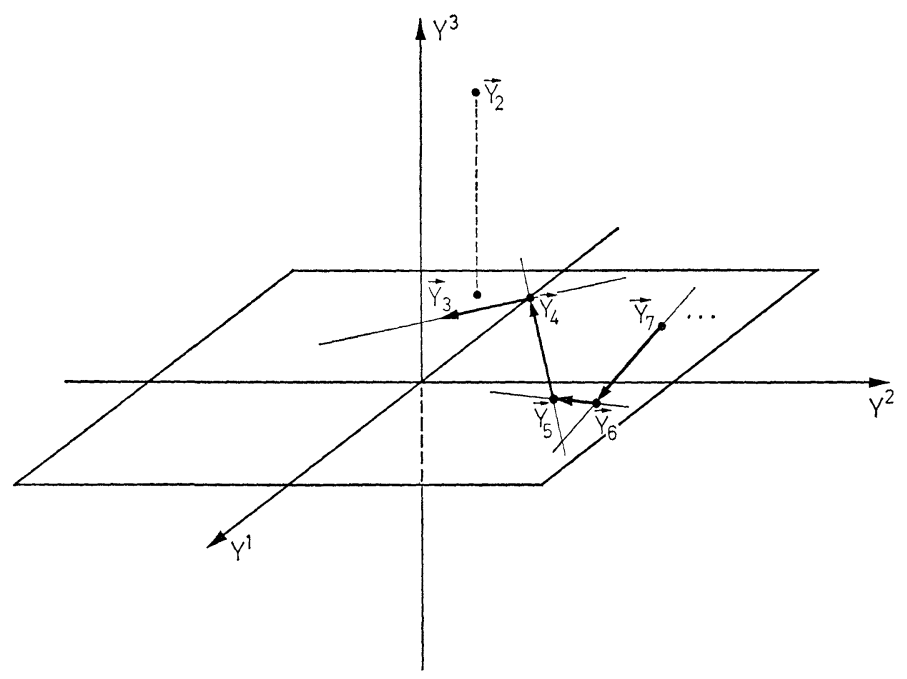

Fig. 4

Dann folgt für alle reellen Punkte $y_{1}, y_{2}, \ldots, y_{n}$ mit $\left(y_{1}-y_{2}\right)^{2}<0$ :

$$
\left\langle\left[A\left(y_{1}\right), A\left(y_{2}\right)\right] A\left(y_{3}\right) \ldots A\left(y_{n}\right)\right\rangle=0 .
$$

Wir wollen den Beweis für den Fall $\gamma_{(n)}\{(y)\} \in$ einem abgeschlossenen Intervall von ] 0, 1 [ führen. Dabei wird auch für die anderen Fälle das Prinzip der Schlußweise klar.

Zum Beweis der Behauptung konstruieren wir unter Berücksichtigung der bisherigen Ergebnisse dieses Abschnitts wiederum eine Funktion, welche die Voraussetzungen des schon erwähnten Theorems über Funktionen von exponentiellem Typ [6] erfüllt - nämlich

$$
\begin{gathered}
\left\{W_{123 \ldots n}^{\mathrm{zykl}}-W_{213 \ldots n}^{\mathrm{zykl}}\right\}\left(-y_{2}+w\left(\begin{array}{l}
0 \\
0 \\
0 \\
1
\end{array}\right), y_{2}-y_{3}-\left[1-\gamma_{(n)}\{(y)\}\right] w\left(\begin{array}{l}
0 \\
0 \\
0 \\
1
\end{array}\right), y_{3}-y_{4},\right. \\
\left.\ldots, y_{n-1}-y_{n}\right)_{w^{\beta}=z},
\end{gathered}
$$

und schließen so: $\left\langle\left[A\left(y_{1}\right), A\left(y_{2}\right)\right] A\left(y_{3}\right) \ldots A\left(y_{n}\right)\right\rangle=0$ auf einer offenen Menge von in den Reihenfolgen $1,2,3, \ldots, n$ und $2,1,3, \ldots, n$ total raumartigen Punkten. Wenden wir nun das Wightmansche Argument [5] an, so folgt wie behauptet für alle reellen Punkte

$y_{1}, y_{2}, \ldots, y_{n}$ mit $\left(y_{1}-y_{2}\right)^{2}<0:\left\langle\left[A\left(y_{1}\right), A\left(y_{2}\right)\right] A\left(y_{3}\right) \ldots A\left(y_{n}\right)\right\rangle=0$.

Nun gibt es für beliebige raumartig getrennte Punkte $x_{1}$ und $x_{2}$ stets eine offene Menge von reellen Punkten, die sowohl in den Reihenfolgen $1,2,3,4, \ldots, n-1, n$ und $n, 1,2,3, \ldots, n-2, n-1$ wie auch in den Reihenfolgen $2,1,3,4, \ldots, n-1, n$ und $n, 2,1,3, \ldots, n-2, n-1$ 
total raumartig sind. In solchen Punkten gilt:

$$
\begin{aligned}
\left\langle A\left(x_{1}\right) A\left(x_{2}\right) A\left(x_{3}\right) A\left(x_{4}\right)\right. & \left.\ldots A\left(x_{n-1}\right) A\left(x_{n}\right)\right\rangle \\
& =\left\langle A\left(x_{2}\right) A\left(x_{1}\right) A\left(x_{3}\right) A\left(x_{4}\right) \ldots A\left(x_{n-1}\right) A\left(x_{n}\right)\right\rangle \\
\left\langle A\left(x_{1}\right) A\left(x_{2}\right) A\left(x_{3}\right) A\left(x_{4}\right)\right. & \left.\ldots A\left(x_{n-1}\right) A\left(x_{n}\right)\right\rangle \\
= & \left\langle A\left(x_{n}\right) A\left(x_{1}\right) A\left(x_{2}\right) A\left(x_{3}\right) \ldots A\left(x_{n-2}\right) A\left(x_{n-1}\right)\right\rangle \\
\left\langle A\left(x_{2}\right) A\left(x_{1}\right) A\left(x_{3}\right) A\left(x_{4}\right)\right. & \left.\ldots A\left(x_{n-1}\right) A\left(x_{n}\right)\right\rangle \\
= & \left\langle A\left(x_{n}\right) A\left(x_{2}\right) A\left(x_{1}\right) A\left(x_{3}\right) \ldots A\left(x_{n-2}\right) A\left(x_{n-1}\right)\right\rangle
\end{aligned}
$$

und daher: $\left\langle A\left(x_{n}\right)\left[A\left(x_{1}\right), A\left(x_{2}\right)\right] A\left(x_{3}\right) \ldots A\left(x_{n-1}\right)\right\rangle=0$.

Nochmalige Anwendung der Wightmanschen Argumentation und Umbenennung liefern:

$\left\langle A\left(x_{1}\right)\left[A\left(x_{2}\right), A\left(x_{3}\right)\right] A\left(x_{4}\right) \ldots A\left(x_{n}\right)\right\rangle=0$ für alle reellen Punkte

$$
x_{1}, \ldots, x_{n} \text { mit }\left(x_{2}-x_{3}\right)^{2}<0 .
$$

Indem wir diese Beweiskette fortsetzen, erhalten wir schließlich

Satz 2. Sei $A(x)$ ein hermitesches, skalares, temperiertes Feld, welches alle Wightman-Axiome erfiillt mit evtl. Ausnahme des Lokalitätsaxioms. Genügt $A(x)$ den Bedingungen $B_{n}(1)$ und $B_{n}(2)$ für alle natürlichen Zahlen n, so ist das Feld $A(x)$ strikt lokal.

\section{Anhang}

Es gibt keine in der Halbebene $\operatorname{Re} z>0$ reguläre, polynomial beschränkte Funktion $f(z) \neq 0$ mit

$$
\limsup _{z \text { reell } \rightarrow+\infty} \frac{\log |f(z)|}{|z|}=-\infty .
$$

Beweis. Ohne Beschränkung der Allgemeinheit dürfen wir annehmen: $f(z)$ ist stetig in $\operatorname{Re} z \geqq 0$ und $|f(i y)|<M$.

Wir definieren: $F_{\omega}(z)=e^{\omega z} \cdot f(z) \quad \omega>0$.

i) $F_{\omega}(z)$ ist in $\operatorname{Re} z>0$ regulär, in $\operatorname{Re} z \geqq 0$ stetig und von exponentiellem Typ.

$$
\left|F_{\omega}(i y)\right|<M .
$$

iii) Es existiert eine Konstante $m_{\omega}>0$, so daß $\left|F_{\omega}(x)\right|<m_{\omega}$ für $x>0$ Aus dem Satz von Phragmen-Lindelöf [4] folgt:

$$
\left|F_{\omega}(z)\right|<\operatorname{Max}\left\{m_{\omega}, M\right\} \quad \text { in } \operatorname{Re} z \geqq 0 .
$$

Daher gilt für $\frac{F_{\omega}(z)}{[z+1]^{2}}$ die Cauchy-Integralformel:

$$
\begin{gathered}
\frac{F_{\omega}(z)}{[z+1]^{2}}=\frac{1}{2 \pi i} i \int_{-\infty}^{+\infty} d y \frac{F_{\omega}(i y)}{[i y+1]^{2}[i y-z]} \\
|f(z)| e^{\omega|z| \cos (\arg z)}=\left|F_{\omega}(z)\right| \leqq \frac{|z+1|^{2}}{2 \cdot|x| \cdot \pi} M \int_{-\infty}^{+\infty} \frac{d y}{1+y^{2}}=\frac{|z+1|^{2}}{2|x|} M .
\end{gathered}
$$


Die rechte Seite dieser Abschätzung ist unabhängig von $\omega$. Daher folgt: $f(z) \equiv 0$ in $\operatorname{Re} z>0$ und wegen der vorausgesetzten Stetigkeit in $\operatorname{Re} z \geqq 0$.

Einer von uns (K. P.) möchte Herrn M. Scheunert für viele nützliche Diskussionen und fruchtbare Kritik danken.

\section{Literatur}

1. Pohlmeyer, K.: Commun. math. Phys. 7, 80 (1968).

2. Jost, R.: Helv. Phys. Acta 30, 409 (1957).

3. Källén, G., and A. S. Wightman : Dan. Vid. Selsk. Mat. Fys. Skr. 1, no 6 (1958).

4. Trtchmarsh, E. C.: The theory of functions chap. 5, sec. 6. Oxford University Press (1952).

5. Streater, R. F., and A. S. Wightman: PCT, spin \& statistics, and all that, p. 134. New York-Amsterdam:W. A. Benjamin, Inc. 1964.

6. Boas, R. P.: Entire functions, chap. 5, sec. 1, theorem 12. New York: Academic Press Inc. 1954.

Dr. K. Pohlineyer

II. Institut für theoretische Physik

der Universität

2000 Hamburg 50

Luruper Chaussee 149 